

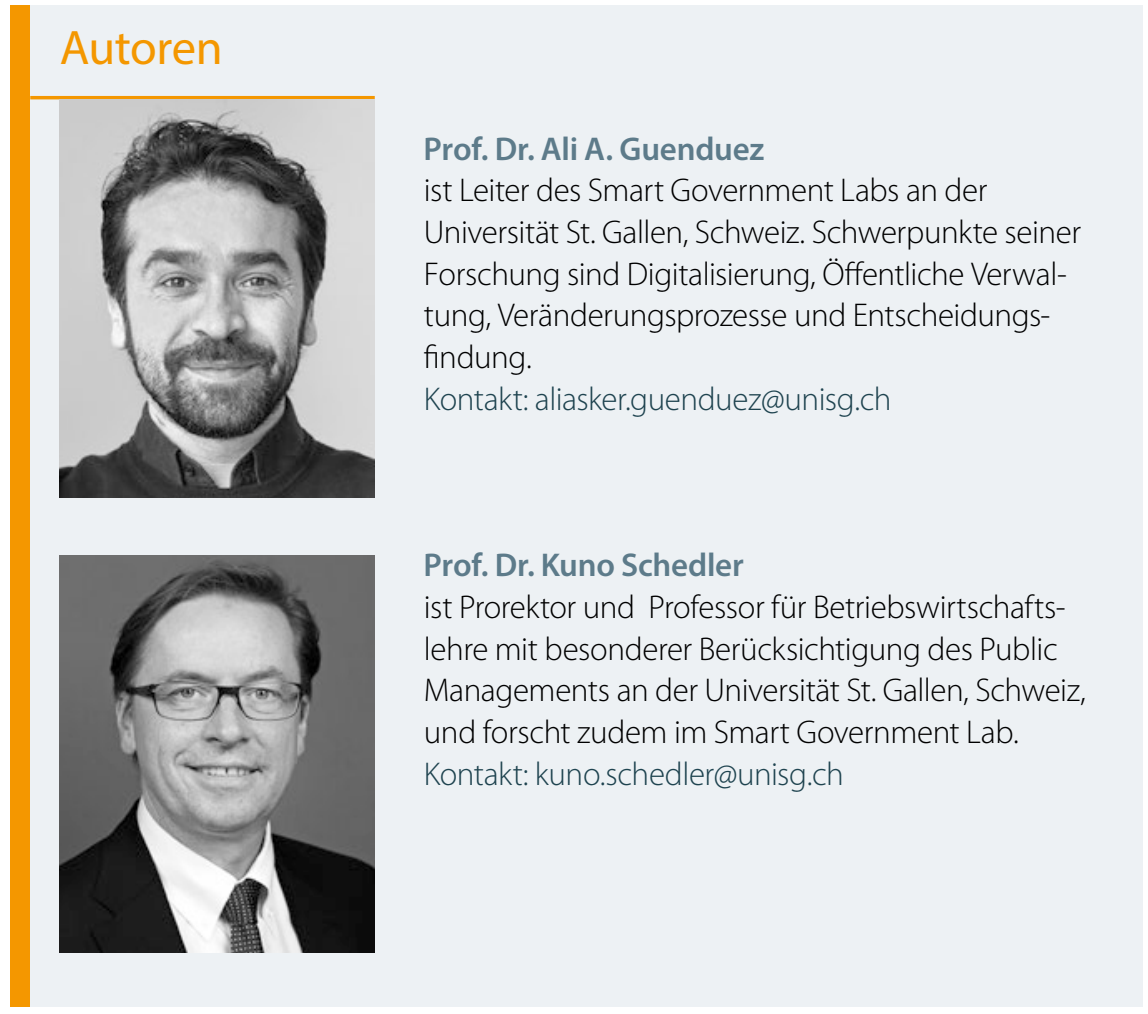

Krankheiten bilden die Grundlage für die Maßnahmen, die heute zur Bekämpfung der Ausbreitung der aktuellen Krankheiten ergriffen werden.

Die italienische Stadt Ferrara entwickelte als Reaktion auf die Pest wirksame Maßnahmen: Isolation, Quarantäne und Hygienevorschriften halfen bereits damals, die Ausbreitung der Epidemie einzudämmen. Die Stadt setzte zwar ihre Handelsaktivitäten mit den betroffenen Regionen fort, hielt aber nur zwei ihrer Tore offen, um den Austausch mit Außenstehenden zu begrenzen. Die Stadt erkannte die Bedeutung eines integrierten Managements der Seuche. So wurden die betroffenen Menschen früh identifiziert. Sie und ihnen nahestehenden Personen wurden isoliert und in Lazaretten außerhalb der Stadt versorgt. Ihre Häuser wurden dekontaminiert, um die Ansteckung einzudämmen. Zudem galt auch schon damals die persönliche Hygiene als ein wichtiges Element zur Abwehr der Krankheit (Vicentini et al., 2020).

In der Stadt Cádiz begann die spanische Regierung zu Beginn des 19. Jahrhunderts mit einer erweiterten Nutzung von Statistiken, insbesondere zur Bekämpfung der Gelbfieber-Epidemie, von der die Stadt aufgrund ihres bedeutenden Handelsstatus besonders betroffen war. Es wurden ein zentrales Informationssystem geschaffen und Berichte über die Zahl der verstorbenen Bürgerinnen und Bürger zusammengetragen. Die Zahl der kranken, geheilten und verstorbenen Personen wurde täglich erfasst, und diese Informationen wurden wöchentlich an die zentrale Stelle gemeldet. Die Zentralisierung der Informationen über die sich ausbreitende Krankheit und der damit gewonnene breitere Überblick über die Ausbreitung des Gelbfiebers half der Regierung, die Krankheit besser zu verstehen (Capelo/ Araújo, 2019).

Gegen Ende des vergangenen Jahrhunderts wurden weitere wertvolle Erkenntnisse über den Umgang mit Epidemien gewonnen. Tarantola und Foster (2011) nennen sechs wichtige Errungenschaften. Erstens ist es wichtig zu verstehen, wie Gemeinschaften funktionieren und was ihre Bedürfnisse sind. Zweitens sollten die Gemeinschaftsstrukturen und -dynamiken analysiert werden, um die Faktoren zu ermitteln, welche die Anfälligkeit für die Verbreitung der Krankheit beeinflussen. Drittens sollten die personelle Kapazität und viertens ihr Wille zur Ausrottung der Epidemie sichergestellt werden. Fünftens sollen der private und der öffentliche Sektor im Kampf gegen eine Epidemie zusammenarbeiten. Schließlich sollten die Bürger aufgefordert werden, ihre allgemeine persönliche Gesundheit zu schützen.

\section{Bekämpfung von Covid-19 in smarten Städten}

In smarten Städten und Regionen werden mit neuen und innovativen Technologien wie etwa Internet of Things (IoT), Sensoren, Überwachungskameras und Wearables Daten gesammelt und anhand Künstlicher Intelligenz (KI) analysiert. $\mathrm{Zu}$ smarten Städten gehören auch neue Formen der Interaktion mit den Bürgerinnen und Bürgern, neue Arbeitsweisen oder neue Modi des Regierens. Die Herausforderungen gleichen jenen aller „Vorgänger-Pandemien“, indem folgende Aufgaben zu lösen sind: 
- Infizierte frühzeitig erkennen

- Medizinische Kapazitäten aufbauen

- Ausbreitung eindämmen

- Staatliche Maßnahmen durchsetzen

- Das Verhalten der Menschen beeinflussen

Dies wird durch die Möglichkeiten des Smart Governments unterstützt. Dabei zeigen sich je nach politischem System sowohl zentrale als auch dezentrale Ansätze: Zentralistisch gelenkte Staaten setzen smarte Technologien ein, um über Top-down-Anweisungen die Bevölkerung zu kontrollieren. Daten werden zentral erhoben, ausgewertet und gespeichert, und nicht selten werden sie zusätzlich für eine obrigkeitliche Überwachung verwendet. In chinesischen Städten kommen KI-gestützte Systeme zum Einsatz, die bis zu 15 Patienten pro Sekunde aus einer Entfernung von maximal drei Metern auf der Grundlage eines berührungslosen Fern-Temperaturscreenings erfassen können. Ein KI-System in Dubai erkennt die Nummernschilder, um Bürgerinnen und Bürger zu identifizieren, die ihre Häuser während einer Abriegelung ohne Genehmigung verlassen (Oxford Business Group, 2020). In verschiedenen Regionen Indiens werden Daten von Kameras und Drohnen zur Überwachung der Bewegungen von Personen und deren Aufenthaltsorten verwendet. Demokratische Systeme setzen hingegen eher auf dezentrale Lösungen mit Eigenverantwortung der Bürgerinnen und Bürger. So speichert etwa die SwissCovid-App keine Daten zentral, sondern alarmiert Benutzerinnen und Benutzer aufgrund in den individuellen Geräten gespeicherter Daten, wenn sie sich länger als 15 Minuten in der Nähe einer infizierten Person aufgehalten haben.

\section{Aktive und passive Partizipation}

Damit die Pandemie erfolgreich bekämpft werden kann, sind die Behörden auf die Mitwirkung der Bürgerinnen und Bürger angewiesen. Diese kann in smarten Städten aktiv und passiv erfolgen
(Guenduez et al., 2017). Aktive Partizipation erfolgt durch die Teilnahme an Programmen, an Abstimmungen und Umfragen. Rückmeldungen zu politischen Interventionen können Entscheidungsfindungsprozesse verbessern sowie die Entwicklung geeigneter Politiken entscheidend beeinflussen. Ein weiteres Beispiel findet sich in verschiedenen Regionen Indiens. Mit der von den Behörden lancierten App müssen Personen in Quarantäne stündlich ein Selfie mit ihrem Standort hochladen. Auf diese Weise stellen die Behörden sicher, dass Durchsetzungsmaßnahmen eingehalten werden. Passive Partizipation erfolgt unter anderem durch das Freischalten eigener Daten, die für die smarte Auswertung durch die Stadt verwendet werden. Als herausragendes Beispiel gelten hier die diversen Covid-Apps, die von den Bürgerinnen und Bürgern auf ihre mobilen Geräte heruntergeladen werden, damit sie relevante Daten erheben und übermitteln können. Standortdaten von Mobiltelefone wurden in mehreren Städten Asiens und des Nahen Ostens verwendet, um Personen zu orten und ihre Kontakte mit anderen zu überwachen.

\section{Pantoffeltierchen-Politik}

Was noch 2017 als eher futuristische Metapher für die neuen Möglichkeiten der Politik beschrieben wurde (Schedler/Gündüz, 2017), ist mit Covid-19 Realität geworden: Echtzeit-Informationen führen zu einer kurzfristigen, experimentellen Pantoffeltierchen-Politik, die starke Züge von „Versuch und Irrtum“Strategien annimmt. Viele Regierungen sind gezwungen, aufgrund schlechter und unvollständiger Information teilweise einschneidende Maßnahmen zu beschließen. Ob sie richtig entschieden haben, zeigt sich erst im Nachhinein (Willi et al., 2020). Zudem kommen laufend neue, aktuelle und qualitativ andere Informationen hinzu, auf die wiederum reagiert werden muss. Regierungen arbeiten mit den größten Telekommunikationsunternehmen zusammen, um mithilfe von aggregierten und anonymisierten Handy-Standortdaten zu eruieren, ob soziale Distanzierungsregeln eingehalten werden. Durch die Kombination von Daten von Kreditund Debitkarten, Mobiltelefonen und Videoüberwachung gelang es der südkoreanischen Regierung, Zeit-Raum-Karten zu erstellen und Interaktionsknotenpunkte zu identifizieren. Insgesamt erweisen sich KI-gestützte Systeme sehr effizient in der Früherkennung (Kummitha, 2020), was politische Reaktionen „in Echtzeit“ ermöglicht und geradezu herausfordert.

Der Aufklärung der Bevölkerung kommt dabei in der Bekämpfung von Pandemien eine besondere Bedeutung zu. Regierungen nutzen verschiedene Kommunikationskanäle, darunter verstärkt auch Soziale Medien, um Updates über Covid-19 zu kommunizieren. In Singapur ist ein Roboterhund im Einsatz, der die Bürgerinnen und Bürger daran erinnert, Abstandsregeln einzuhalten (Oxford Business Group, 2020).

Diese agile Führung der Bevölkerung ist für die Regierungen aber auch eine Herausforderung. In der Schweiz tagte der Bundesrat in den intensivsten Krisenwochen bis zu zweimal wöchentlich, um die Bevölkerung über Maßnahmen gegen Covid-19 zu informieren. Dies bedeutete, dass sich die Maßnahmen rasch änderten, die Informationen über die Zeit widersprüchlich waren und - nicht zuletzt auch bei Städten und Kantonen - Verwirrung entstand. Es zeigt sich, dass der Agilität einer nationalen Politik in solchen Situationen auch Grenzen gesetzt sind. Umso wichtiger ist es für die Regierung, das Vertrauen der Bürgerinnen und Bürger zu gewinnen.

\section{Ökosysteme der sozialen Marktwirtschaft}

Die Zusammenarbeit zwischen verschiedenen Akteurinnen und Akteuren aus dem privaten und dem öffentlichen Sektor hat auch bei Covid-19 Bedeutung, und neue Modelle der Leistungserbrin- 
gung sind zu beobachten (Schedler/Bolz, 2020). Das Schweizer Modell der raschen Hilfe für Unternehmen arbeitet mit einem innovativen Geschäftsmodell: Private Banken vergeben unkompliziert und rasch zinsfreie Kredite bis 500.000 Schweizer Franken, der Bund übernimmt die Garantie. Damit werden zwei zentrale Fähigkeiten der beteiligten Akteure kombiniert und in einem Ökosystem von Banken, Unternehmen und Staat verbunden. Auch die Entwicklung von Covid-Apps entsteht in engen Kooperationen zwischen Behörden, Forschungsinstituten, privaten Firmen und Start-ups.

\section{Fazit}

Im Rahmen der Bekämpfung der aktuellen Pandemie werden viele Maßnahmen, die in den vergangenen Jahrhunderten eingeführt wurden, auch heute noch genutzt. Die Maßnahmen werden jedoch durch den Einsatz von smarten Technologien und Daten optimiert. Daten und Künstliche Intelligenz helfen dabei, das menschliche Verhalten besser zu verstehen, ermöglichen eine schnelle und wirksame Ermittlung von Kontaktpersonen, zeigen, wie effektiv die Maßnahmen sind, lassen smarte Überwachungssysteme entstehen, machen eine Rückverfolgung der Entwicklung der Pandemie möglich und lassen Orte auf der Karte erkennen, die eine Verbreitung begünstigen. Aktive und passive Partizipation der Bürgerinnen und Bürger sind für den Erfolg entscheidend, und diese bedingen Vertrauen.

\section{Literatur}

Capelo M., Araújo P. (2019): Contabilidad ad hoc para la gestión municipal de una crisis epidémica: la fiebre amarilla en Cádiz en 1800, in: De Computis - Revista Española de Historia de la Contabilidad, 16 (2), 42-68.

Guenduez, A. A. Mettler, T., Schedler, K. (2017): Smart Government - Partizipation und Empowerment der Bürger im Zeitalter von Big Data und personalisierter Algorithmen, in: HMD - Praxis der Wirtschaftsinformatik, 4, 477-487, www.springerprofessional.de/link/12175712

Oxford Business Group (2020). What is the future for smart cities after Covid-19? Retrieved from Oxford Business Group: https://oxfordbusinessgroup.com/news/what-future-smart-cities-after-covid-19 (Abruf am 16.06.2020).

Rama Krishna Reddy Kummitha (2020): Smart technologies for fighting pandemics: The techno- and human- driven approaches in controlling the virus transmission, in: Government Information Quarterly, https://doi.org/10.1016/j. giq.2020.101481

Schedler, K., Bolz, U. (2020), Innovative Geschäftsmodelle: Staat und Wirtschaft, in: Swiss Yearbook of Administrative Sciences, 11(1), S. 25-37.

Schedler, K., Gündüz, A. A. (2017): Pantoffeltierchen-Politik dank Echtzeit-Information, in: innovative Verwaltung, 4, S. 10-13, www.springerprofessional.de/link/12209780

Tarantola, D., Foster, S. O. (2011): From smallpox eradication to contemporary global health initiatives: Enhancing human capacity towards a global public health goal, in: Vaccine, 29 (Supplement 4), S. 135-140.

Vicentini, C. B., Manfredini, S., Mares, D., Bonacci, T., Scapoli, C., Chicca, M., Pezzi, M. (2020): Empirical "integrated disease management" in Ferrara during the Italian plague (1629-1631), in: Parasitology International, 75, 102046.

\section{SpringerProfessional.de}

Pantoffeltierchen-Politik

Schedler, K., Guenduez, Ali A. (2017): Pantoffeltierchen-Poltik dank Echtzeit-Information, in: innovative Verwaltung, 4,

S. 10-13, www.springerprofessional.de/link/ 12209780

\section{9 vorbildliche Beispiele für neue Wege in der Wirtschaftsförderung \\ J. Stember (Hrsg.) \\ Innovative Wirtschaftsförderungen \\ in Deutschland \\ - Innovative Strategien erfolgreiche Praxisbeispiele von Städten, Landkreise und Regionen \\ - In dieser Zusammenstellung bislang einmalig}

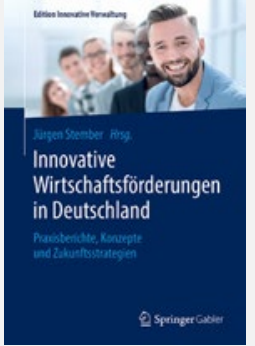

Praxisberichte, Konzepte und

Zukunftsstrategien

2020. X, 366 S. 127 Abb., 5 Abb. in Farbe. Brosch.

$€$ (D) $39,99 \mid €$ (A) 41,11 | CHF 44.50

ISBN 978-3-658-28482-4

$€$ (D) 29,99 | CHF 35.50

ISBN 978-3-658-28483-1 (eBook)
Dieses Buch stellt in 49 ausgezeichneten Projekten den Ideenreichtum der Wirtschaftsförderung auf kommunaler Ebene in Deutschland vor. Damit ist dieses Buch eine wahre Schatztruhe für Praktiker in der Wirtschaftsförderung sowie Wissenschaftler und Studierende. Die Organisatoren des "Forum deutscher Wirtschaftsförderer" und die Hochschule Harz haben in 2019 erstmals einen bundesweiten Award "Innovative Wirtschaftsförderungen" ausgelobt und alle kommunalen, kreisweiten und regionalen $[\ldots]$ 